\title{
Construction of a prognostic prediction system for pancreatic ductal adenocarcinoma to investigate the key prognostic genes
}

\author{
BINGLI ZHENG ${ }^{1}$, JIE PENG $^{2}$, ABLIKIM MOLLAYUP $^{1}$, AHMAT BAKRI $^{1}$, \\ LEI GUO ${ }^{1}$, JIANJIANG ZHENG ${ }^{1}$ and HUI XU ${ }^{1}$ \\ ${ }^{1}$ Department of Pancreatic Surgery, Xinjiang Uygur Autonomous Region People's Hospital, Ürümqi, \\ Xinjiang 830002; ${ }^{2}$ Emergency Department, Traditional Chinese Medicine Hospital of \\ Xinjiang Medical University, Ürümqi, Xinjiang 830000, P.R. China
}

Received May 4, 2017; Accepted September 19, 2017

DOI: $10.3892 / \mathrm{mmr} .2017 .7850$

\begin{abstract}
Pancreatic cancer (PC) is associated with high mortality rates and poor prognoses. Pancreatic adenocarcinoma is the most common type of $\mathrm{PC}$, and almost all cases of pancreatic adenocarcinoma are pancreatic ductal adenocarcinoma (PDAC). The aim of the current study was to reveal the genes involved in the prognosis of PDAC. Five datasets, including GSE71729 (145 PDAC samples and 46 normal samples), GSE15471 (39 PDAC samples and 39 normal samples), GSE1542 (24 PDAC samples and 25 normal samples), GSE28735 (45 PDAC samples and 45 normal samples) and GSE62452 (69 PDAC samples and 69 normal samples) were downloaded from the Gene Expression Omnibus database. Using the MetaDE.ES method in the MetaDE package, differentially expressed genes (DEGs) were identified from the five datasets. Furthermore, prognosis-associated genes were screened using the Cox regression analysis in the survival package, and co-expression network and module analyses were performed separately using Cytoscape software and GraphWeb tool, respectively. After a prognostic prediction system was constructed and validated, enrichment analysis of the signature genes was performed using the clusterProfiler package. A total of 480 DEGs were identified from the five datasets and 259 prognosis-associated genes were screened from GSE28735 and GSE62452. In addition, the prognostic prediction system composed of 67 signature genes [including basic transcription factor 3 (BTF3), serine/threonine kinase 11 (STK11), thrombospondin 1 (THBS1), ribosomal protein L38 (RPL38) and secretin receptor $(S C T R)]$ was constructed and validated. The signature genes involved in the co-expression network were enriched in five pathways. In particular, STK11 was involved
\end{abstract}

Correspondence to: Dr Hui Xu, Department of Pancreatic Surgery, Xinjiang Uygur Autonomous Region People's Hospital, 120 Longquan Street, Ürümqi, Xinjiang 830002, P.R. China E-mail: zhengbingli168@sina.com

Key words: pancreatic ductal adenocarcinoma, prognosis-associated genes, co-expression network, prognostic prediction system, signature genes in three signaling pathways, and THBS1 was enriched in the phosphoinositide 3-kinase-Akt signaling pathway. Thus, BTF3, STK11, THBS1, RPL38 and SCTR may influence the prognosis of PDAC.

\section{Introduction}

Pancreatic cancer (PC), which arises when pancreatic cells proliferate abnormally, is characterized by yellow skin, weight loss, back or abdominal pain, dark urine, light-colored stools, and appetite loss (1). The early symptoms of PC are not obvious, thus newly diagnosed PC cases have usually reached an advanced stage (2). PC is primarily induced by tobacco smoking, diabetes, obesity and genetic conditions $(3,4)$. Globally, PC is the seventh leading cause of cancer-associated mortality, resulting in 330,000 fatalities in 2012 (5). The prognosis of PC is usually very poor, with $5 \%$ of people surviving for five years and $25 \%$ surviving just one year after diagnosis $(5,6)$. Pancreatic adenocarcinoma is the most common type of PC and accounts for $85 \%$ of all PC cases (3). Almost all cases of pancreatic adenocarcinoma originate from the ducts of the pancreas, and are termed pancreatic ductal adenocarcinoma (PDAC) (7). Therefore, revealing the underlying mechanisms of PDAC is significant for developing novel treatments.

Yamazaki et al (8) demonstrated that SMAD family member 3 contributes to the malignant potential of PDAC by inducing epithelial-mesenchymal transition (EMT) in tumor cells and thus presents as a promising biomarker of poor prognosis (8). Masugi et al (9) identified that integrin $\beta 4$ functions in regulating EMT and cancer invasion, and its overexpression has clinicopathological and prognostic significance in PDAC (9). Sex determining region Y-box 9 (SOX9) and phosphorylated-v-Akt murine thymoma viral oncogene homolog 1 $(p-A K T)$ are reported to be associated with proliferation and distant metastasis, indicating that $S O X 9$ and $p-A K T$ may potentially predict the prognosis of PDAC (10). The downregulation of anterior gradient 2 is induced by EMT and serves as a novel prognostic marker in patients with PDAC (11). In PDAC patients who undergo pancreaticoduodenectomy, high expression levels of transforming growth factor $\beta-1$ may inhibit the poorer survival that is associated with high proliferation (12). 
However, the genes involved in the prognosis of PDAC have not been fully reported.

In the current study, public databases were searched for microarray datasets associated with PDAC in homo sapiens. Subsequently, comprehensive bioinformatics analyses [including identification of differentially expressed genes (DEGs) and prognosis-associated genes, co-expression network and module analyses, construction and validation of prognostic prediction system, and enrichment analysis] were performed successively to investigate the key prognosis-associated genes in PDAC. The current study may contribute to developing targeted therapeutic strategies for improving the prognosis of PDAC.

\section{Data and methods}

Expression profile data. Using 'Pancreatic ductal adenocarcinoma' and 'homo sapiens' as keywords, the gene expression profiles in the Gene Expression Omnibus (GEO; http://www.ncbi.nlm.nih.gov/geo/) database were searched. The datasets that met the following criteria were included: i) The dataset was associated with PDAC; ii) the dataset included PDAC tissue samples and normal control samples. Finally, a total of five datasets were included in the current study, including GSE71729 (based on the GPL20769 platform, including 145 PDAC samples and 46 normal samples), GSE15471 (based on the GPL570 platform, including 39 PDAC samples and 39 normal samples), GSE1542 (based on the GPL96 platform, including 24 PDAC samples and 25 normal samples), GSE28735 (based on the GPL9644 platform, including 45 PDAC samples and 45 normal samples) and GSE62452 (based on the GPL9644 platform, including 69 PDAC samples and 69 normal samples).

Data preprocessing. Using the Affy package (http://www. bioconductor.org/packages/release/bioc/html/affy.html) (13) in $\mathrm{R}$ language, background correction and normalization of raw data of GSE15471 and GSE1542 were conducted. For the datasets of GSE71729, GSE28735 and GSE62452, probes were corresponded to gene symbols based upon platform annotation information. After the unloaded probes were removed, the average value of the probes mapped to the same gene was obtained as the initial gene expression value. Finally, the data were normalized by the linear models for microarray data using the limma package (http://www.bioconductor.org/packages/release/bioc/html/limma.html) (14) in R language.

Meta-analysis to screen characteristic factors. Using the quality control standards [including external quality control, internal quality control, consistency quality control (CQCg and CQCp) and accuracy quality control (AQCg and AQCp)] in the MetaQC package (15), quality control was performed for the datasets. To identify reliable datasets, the datasets were further assessed and screened using the two-dimensional diagram of principal component analysis (PCA) and standardized mean rank scores. Based on the MetaDE.ES method in the MetaDE package (15), homogeneous unbiased genes were identified using a heterogeneity test [thresholds: $\mathrm{Tau}^{2}=0$ and Qpval (statistical parameter representing heterogeneity of the dataset) $>0.05$ ] and the DEGs were screened [threshold: False discovery rate $(\mathrm{FDR})<0.05]$.

Identification of prognosis-associated genes. Among the included datasets, GSE28735 and GSE62452 were based upon the GPL9644 platform and contained sample survival information. Thus, the data of the two datasets were merged and prognosis-associated genes were identified using the Cox regression analysis in survival package, with $\mathrm{P}<0.05$ set as the significant threshold (https://github.com/therneau/survival). After significant P-values were obtained using the log-rank test, Kaplan-Meier (KM) survival analysis was performed on the top six genes with higher-logRank (P-values) (16).

Co-expression network and module analyses. The expression values of the prognosis-associated genes were extracted from the datasets and the COR function (17) in R language was used to calculate their correlation coefficients. The co-expression pairs with correlation coefficient $|r| \geq 0.6$ and $\mathrm{P}<0.05$ were selected for constructing a co-expression network using Cytoscape software (version 3.5.0; http://www.cytoscape.org/) (18). Furthermore, module analysis was performed for the co-expression network using the GraphWeb tool (http://biit.cs.ut.ee/graphweb/) (19).

Construction of a prediction and discrimination system for prognosis. The PDAC samples in GSE28735 and GSE62452 had survival information, and thus were taken as the training dataset for the prediction and discrimination system of prognosis. Firstly, the samples were divided into alive and deceased groups according to their survival states. Secondly, the samples were further divided into groups with bad prognosis (deceased and alive survival time $<15$ months) and good prognosis (alive and alive survival time $\geq 15$ months). After the genes in the co-expression network were sorted in descending order according to their-logRank (P-values), Baye's discriminant analysis was performed using the discriminant.bayes function in the e1071 package (20). Genes were added one by one, and the genes affecting discrimination accuracy were removed until the highest discrimination accuracy was obtained. Under the highest discrimination accuracy, the discrimination coefficient of each sample, gene combination, and discrimination system were defined as the prognostic score, signature gene and prognostic prediction system, respectively.

Validation of the prognostic prediction system. To detect the effect of the prognostic prediction system, KM survival analysis (16) was performed for GSE28735 and GSE62452 to verify the correlation between the sample types recognized by the prognostic prediction system, and the actual survival time and states. In addition, the microarray data under E-MEXP-2780 (downloaded in January 5, 2017; including 30 PDAC samples containing survival information) were downloaded from the European Bioinformatics Institute (http://www.ebi.ac.uk/) database and used as an independent validation dataset for the prognostic prediction system. In addition, the PDAC dataset (downloaded in January 5, 2017; based on Illumina HiSeq 2000 RNA Sequencing platform; including 183 PDAC samples, among which 163 samples had survival information) in The Cancer Genome Atlas (TCGA; https://cancergenome.nih.gov/) database was also downloaded. 
Table I. Results of quality control measures and SMRs.

Dataset IQC EQC CQCg CQCp AQCg AQCp SMR

$\begin{array}{lrrrrrrr}\text { GSE71729 } & 3.85 & 4.83 & 264.65 & 133.86 & 32.71 & 82.01 & 2.42 \\ \text { GSE15471 } & 4.96 & 3.09 & 275.15 & 70.62 & 39.49 & 39.38 & 3.18 \\ \text { GSE1542 } & 4.09 & 4.34 & 242.36 & 103.51 & 101.54 & 58.61 & 3.92 \\ \text { GSE28735 } & 5.11 & 3.21 & 310.21 & 90.31 & 18.24 & 29.18 & 3.17 \\ \text { GSE62452 } & 5.42 & 3.23 & 307.54 & 75.54 & 19.03 & 29.94 & 3.08\end{array}$

IQC, internal quality control; EQC, external quality control; $\mathrm{CQCg}$, consistency quality control for genes; CQCp, consistency quality control for pathways; $\mathrm{AQCg}$, accuracy quality control for genes; AQCp, accuracy quality control for pathways; SMR, standardized mean rank.

Subsequently, the expression values of the signature genes were extracted from E-MEXP-2780 and the PDAC dataset was downloaded from the TCGA database. After prognostic scores were obtained based on the prognostic prediction system, the samples in E-MEXP-2780 and the PDAC dataset downloaded from the TCGA database were divided into groups with bad prognosis and good prognosis. Finally, KM survival analysis (16) was performed to analyze the correlation between the sample types recognized by the prognostic prediction system, and the actual survival time and states.

Construction of the co-expression network for the signature genes and identification of the key genes. The co-expression pairs of the signature genes were identified from the above co-expression network, and the co-expression networks for the signature genes were visualized. Gene Ontology (GO) (21) functional analysis and Kyoto Encyclopedia of Genes and Genomes (KEGG) pathway (22) enrichment analysis were performed on genes in the co-expression network using the clusterProfiler package (http://bioconductor. org/packages/release/bioc/html/clusterProfiler.html) (23) in R. To further identify the key genes associated with PDAC, the enriched terms were integrated into the co-expression network and the genes involved in multiple terms were selected.

\section{Results}

Identification of characteristic factors and prognosis-associated genes. After the raw data of the five datasets were normalized, quality control was further performed for the datasets (Fig. 1; Table I). The two-dimensional diagram of PCA for the five datasets is presented in Fig. 2. As a result, all five datasets were in accordance with the quality control standards and were included for the subsequent analysis. Based on the MetaDE.ES method, a total of 480 DEGs were identified from the five datasets.

GSE28735 and GSE62452 were merged and a total of 259 prognosis-associated genes were screened. Subsequently, KM survival analysis was performed with the top six prognosis-associated genes, including transketolase (TKT), basic transcription factor 3, (BTF3), myomesin 1 (MYOM1), neurexophilin 4 (NXPH4); serine/threonine kinase 24

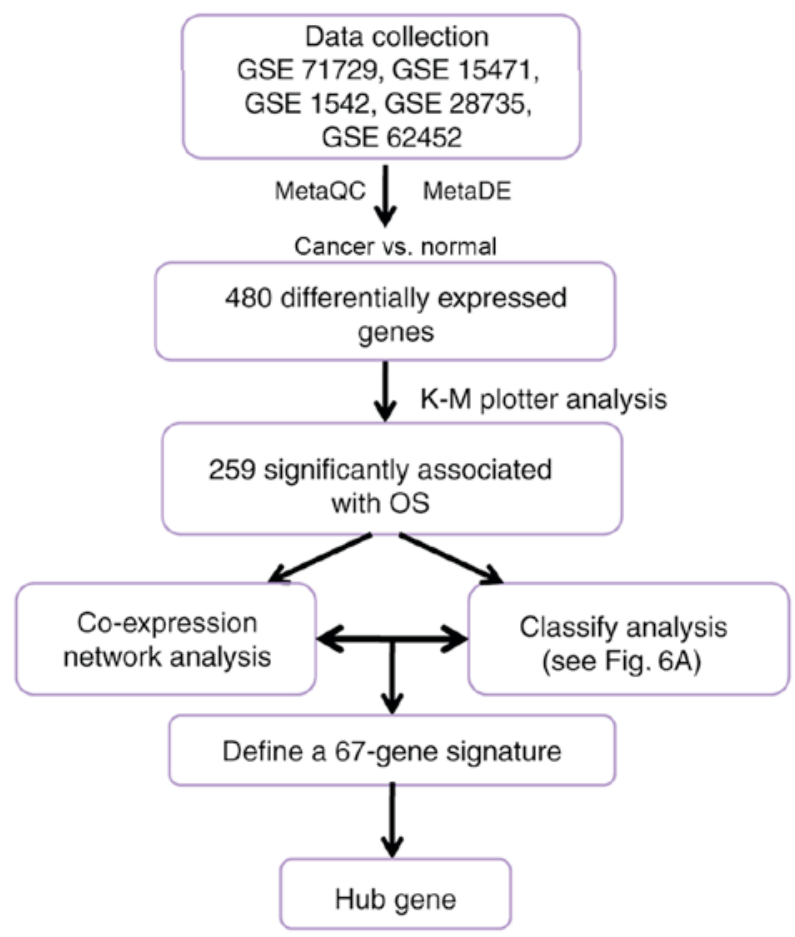

Figure 1. Data analysis flow diagram.

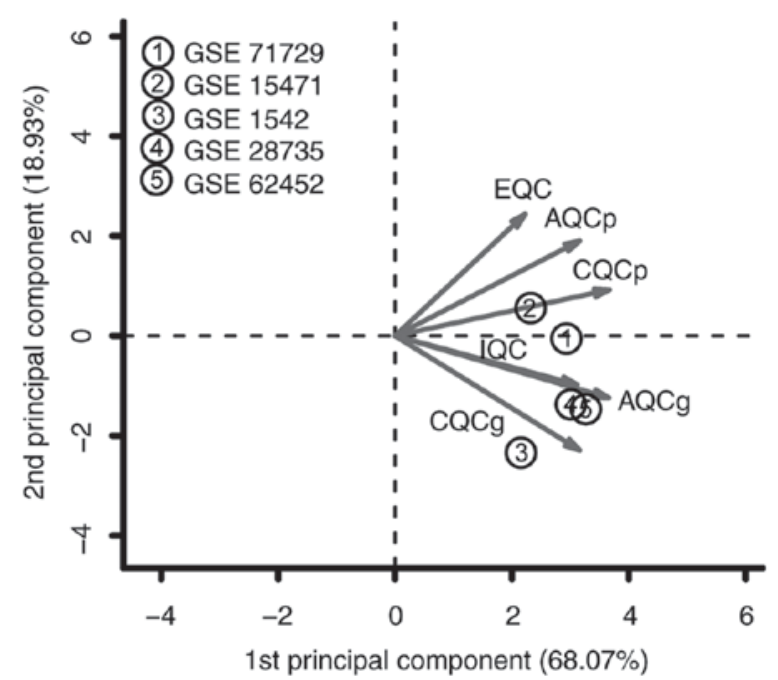

Figure 2. Two-dimensional diagram of principal component analysis for the five datasets. EQC, external quality control; AQCp, accuracy quality control for pathways; CQCp, consistency quality control for pathways; IQC, internal quality control; AQCg, accuracy quality control for genes; CQCg, consistency quality control for genes.

(STK24), transducin $\beta$-like 3 (TBL3) with the highest-logRank (P-values). The KM survival curves are presented in Fig. 3.

Co-expression network and module analyses. Under $|r| \geq 0.6$ and $\mathrm{P}<0.05$, the co-expression pairs among the prognosis-associated genes were identified and the co-expression network (which had 213 nodes and 1,984 edges) was constructed (Fig. 4). Subsequently, a total of six modules were identified from the co-expression network. Furthermore, GO functional enrichment analysis was performed for the genes involved in each of the modules. Additionally, the 

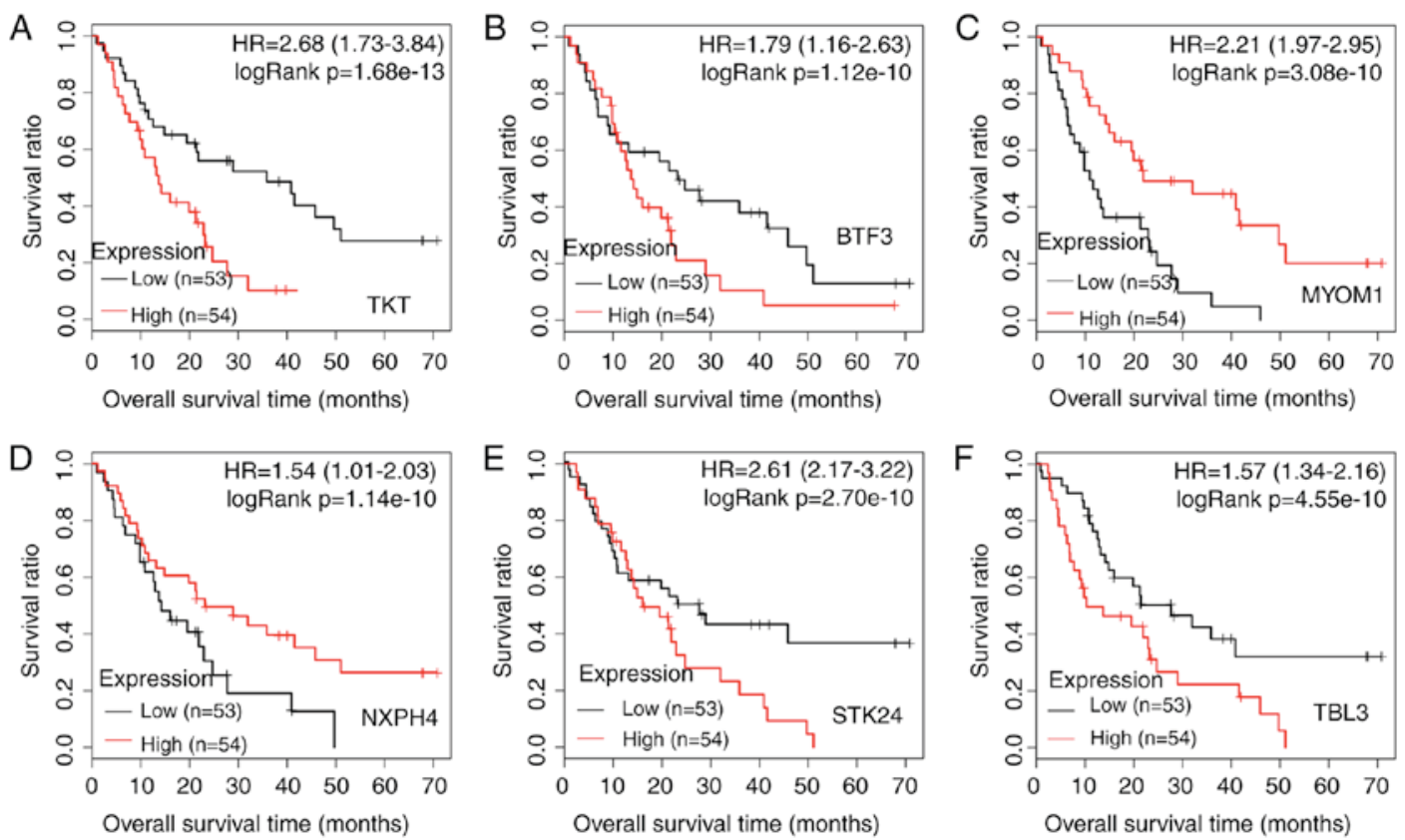

Figure 3. Kaplan-Meier survival curves for the top six prognosis-associated genes with higher-logRank (P-values): (A) TKT, (B) BTF3, (C) MYOM1, (D) NXPH4, (E) STK24 and (F) TBL3. The red and black lines represent samples with high and low expression levels, respectively. HR, hazard ratio; TKT, transketolase; BTF3, basic transcription factor 3; MYOM1, myomesin 1; NXPH4, neurexophilin 4; STK24, Serine/threonine-protein kinase 24; TBL3, transducin $\beta$-like protein 3.

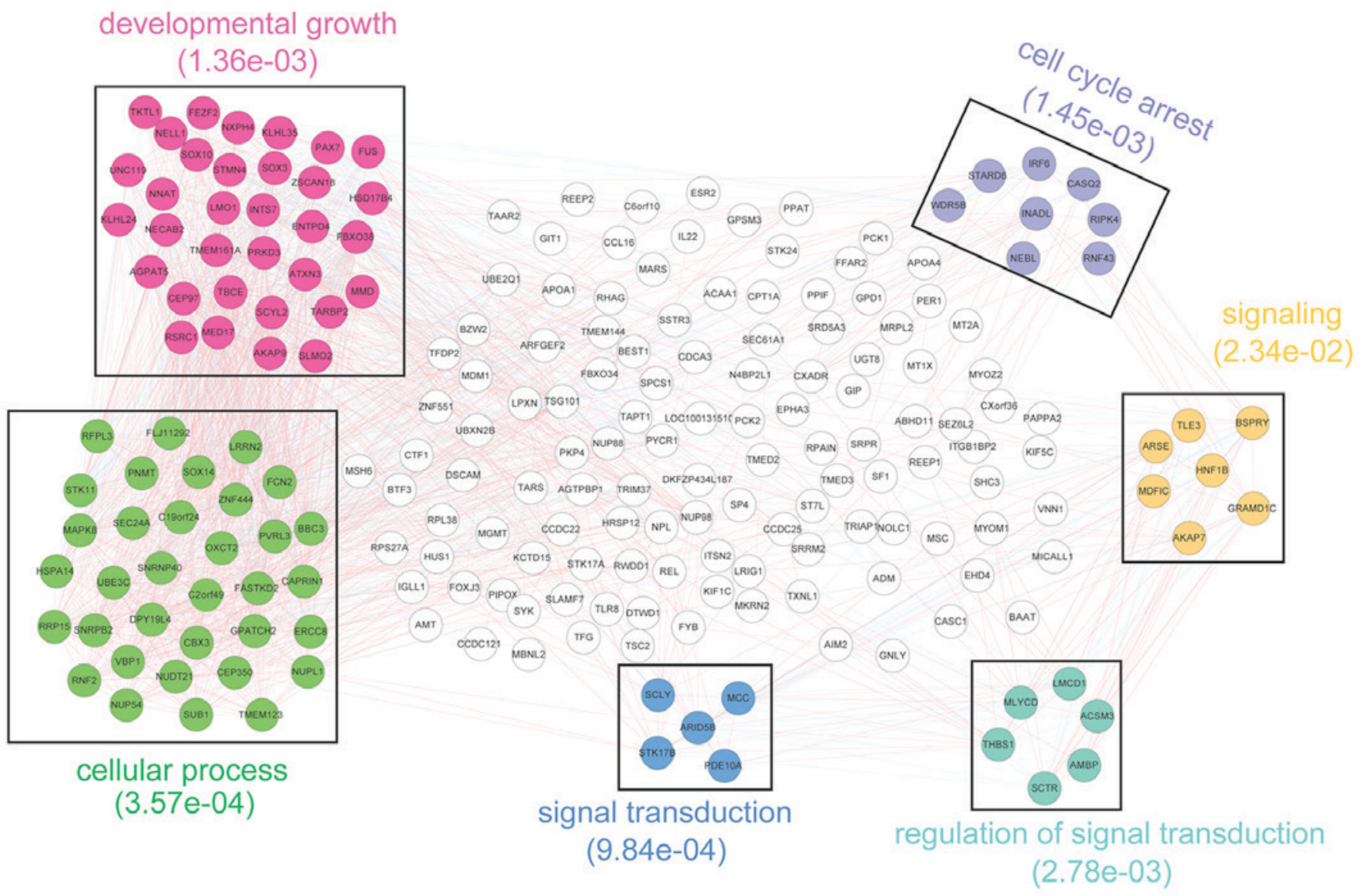

Figure 4. Co-expression network constructed for the prognosis-associated genes. Red and blue lines indicate that correlation coefficients were positive and negative, respectively. The sections within the black squares are modules identified from the network, and the most significant Gene Ontology terms enriched for it are noted in the diagram.

most significant GO terms enriched for purple, yellow, cyan, blue, green, and red modules separately were cell cycle arrest
$(\mathrm{P}=1.45 \mathrm{e}-03)$, signaling $(\mathrm{P}=2.34 \mathrm{e}-02)$, regulation of signal transduction $(\mathrm{P}=2.78 \mathrm{e}-03)$, signal transduction $(\mathrm{P}=9.84 \mathrm{e}-04)$, 
A

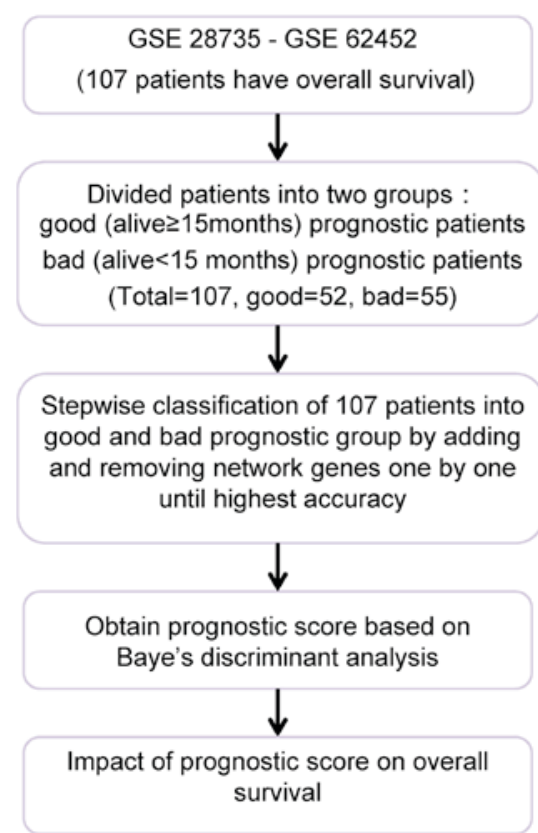

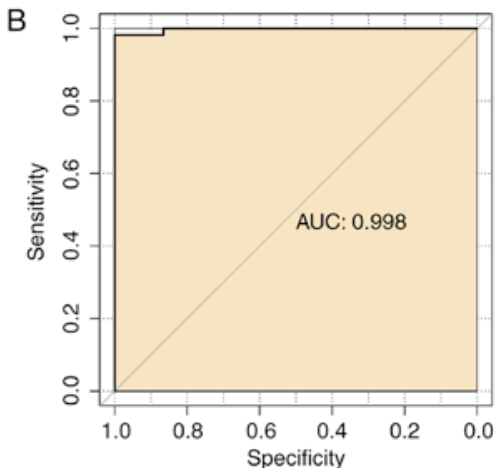

C

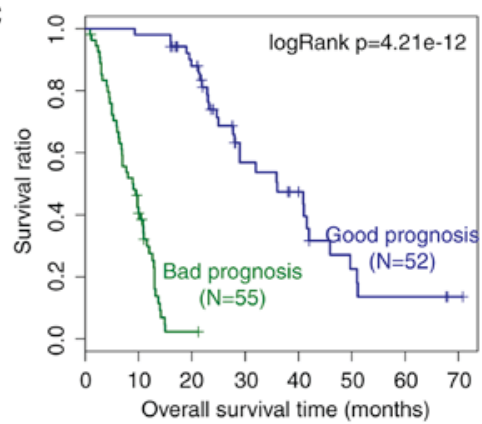

Figure 5. (A) Process for constructing a prognostic prediction system based upon the GSE28735 and GSE62452 datasets. (B) AUC showing the discriminant accuracy of the prognostic prediction system. (C) The Kaplan-Meier survival curve for detecting the effect of the prognostic prediction system based upon GSE28735 and GSE62452. AUC, area under the receiver operating characteristic curve.

cellular process $(\mathrm{P}=3.57 \mathrm{e}-04)$, and developmental growth $(\mathrm{P}=1.36 \mathrm{e}-03)$.

Construction and validation of a prognostic prediction system. A total of 107 PDAC samples in GSE28735 and GSE62452 had survival information, and were divided into groups with good (52 samples) and bad (55 samples) prognoses. According to the process in Fig. 5A, the prognostic prediction system composed of 67 signature genes [including $B T F 3$, serine/threonine kinase 11 (STK11), thrombospondin 1 (THBS1), ribosomal protein L38 (RPL38) and secretin receptor, $(S C T R)]$ was finally constructed. The area under the receiver operating characteristic (ROC) curve (AUC) demonstrating the discriminant accuracy of the prognostic prediction system is presented in Fig. 5B. In addition, a discriminant scoring system was constructed for the prognostic prediction system, which was as follows:

Prognostic score $=a_{i=1}^{67}\left(\right.$ Bayes $^{\prime}$ discriminant analysis $)=\left(\begin{array}{c}{[0,3] \sim \text { bad }} \\ {[-3,0) \sim \text { good }}\end{array}\right)$

KM survival analysis was performed for GSE28735 and GSE62452 to detect the effect of the prognostic prediction system, identifying that the group with good prognosis had a significantly higher survival rate when compared with the group with bad prognosis ( $\mathrm{P}=4.21 \mathrm{e}-12$; Fig. 5C). In addition, the PDAC dataset downloaded from the TCGA database served as an independent validation dataset for the prognostic prediction system. The AUC and KM survival curve in Fig. 6A demonstrates that the survival rate of the good prognosis group was significantly higher than that of the bad prognosis group $(\mathrm{P}=3.17 \mathrm{e}-08)$. Furthermore, the microarray data of E-MEXP-2780 was used to validate the prognostic prediction system, identifying that the group with good prognosis exhibited a significantly higher survival rate when compared with the bad prognosis group $(\mathrm{P}=1.58 \mathrm{e}-06$; Fig. 6B). Therefore, the prognostic prediction system could accurately classify the samples from prognostic level.

Construction of co-expression network for the signature genes and identification of the key genes. The co-expression network constructed for the signature genes included 56 signature genes and 237 edges (Fig. 7). A total of 14 GO_biological process (BP) terms and five KEGG signaling pathways were enriched for the genes involved in the co-expression network (Table II). Meanwhile, the five signaling pathways were merged into the co-expression network for the signature genes. As presented in Fig. 7, phosphoenolpyruvate carboxykinase 1 ( $P C K 1)$ was enriched in all five signaling pathways, and STK11 was involved in three signaling pathways. In addition, THBSI was enriched in the phosphoinositide 3-kinase (PI3K)-Akt signaling pathway $(\mathrm{P}=0.017456)$. In addition, $\mathrm{KM}$ survival analysis was performed for STK11 (Fig. 8A) and PCK1 (Fig. 8B) based on the GSE28735 and GSE62452 datasets.

\section{Discussion}

In the current study, a total of 480 DEGs were identified from five datasets. In addition, 259 prognosis-associated genes were screened from GSE28735 and GSE62452, and BTF3 was among the top six prognosis-associated genes. Furthermore, a total of six modules were identified from the co-expression network for the prognosis-associated genes. A prognostic prediction system composed of 67 signature genes, which included BTF3, STK11, THBS1, RPL38 and SCTR, was finally constructed and validated. Finally, the co-expression network for the signature genes was constructed and the signature genes involved in the co-expression network were enriched in 
A

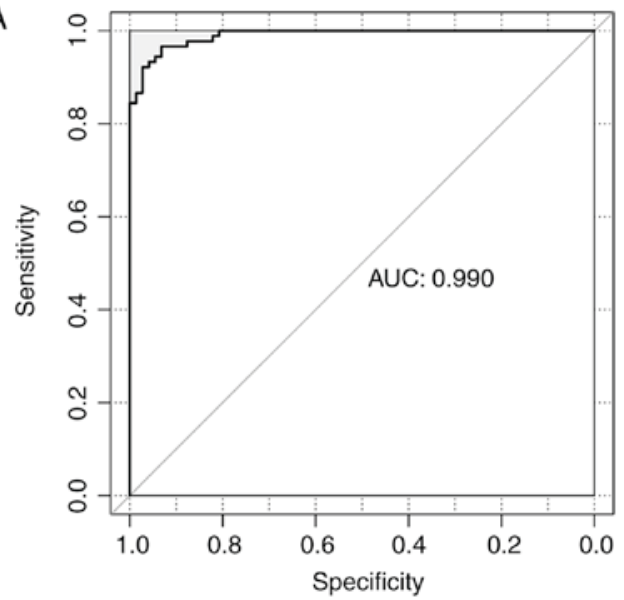

B

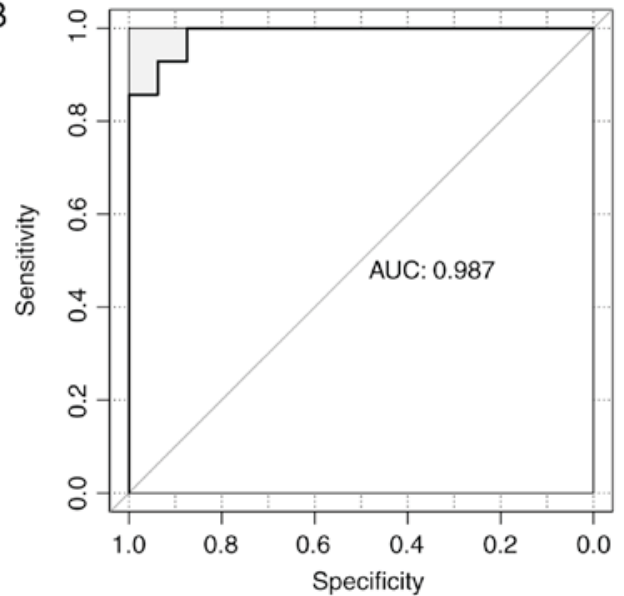

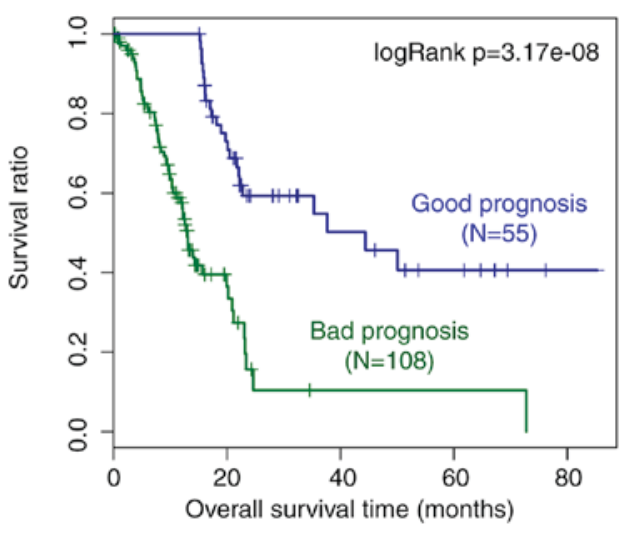

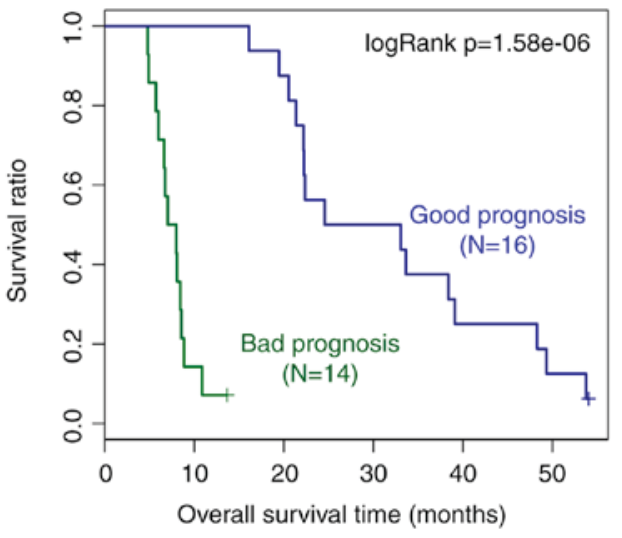

Figure 6. AUC and Kaplan-Meier survival curves for the pancreatic ductal adenocarcinoma dataset downloaded from (A) The Cancer Genome Atlas database and (B) the microarray data of E-MEXP-2780. AUC, area under the receiver operating characteristic curve.

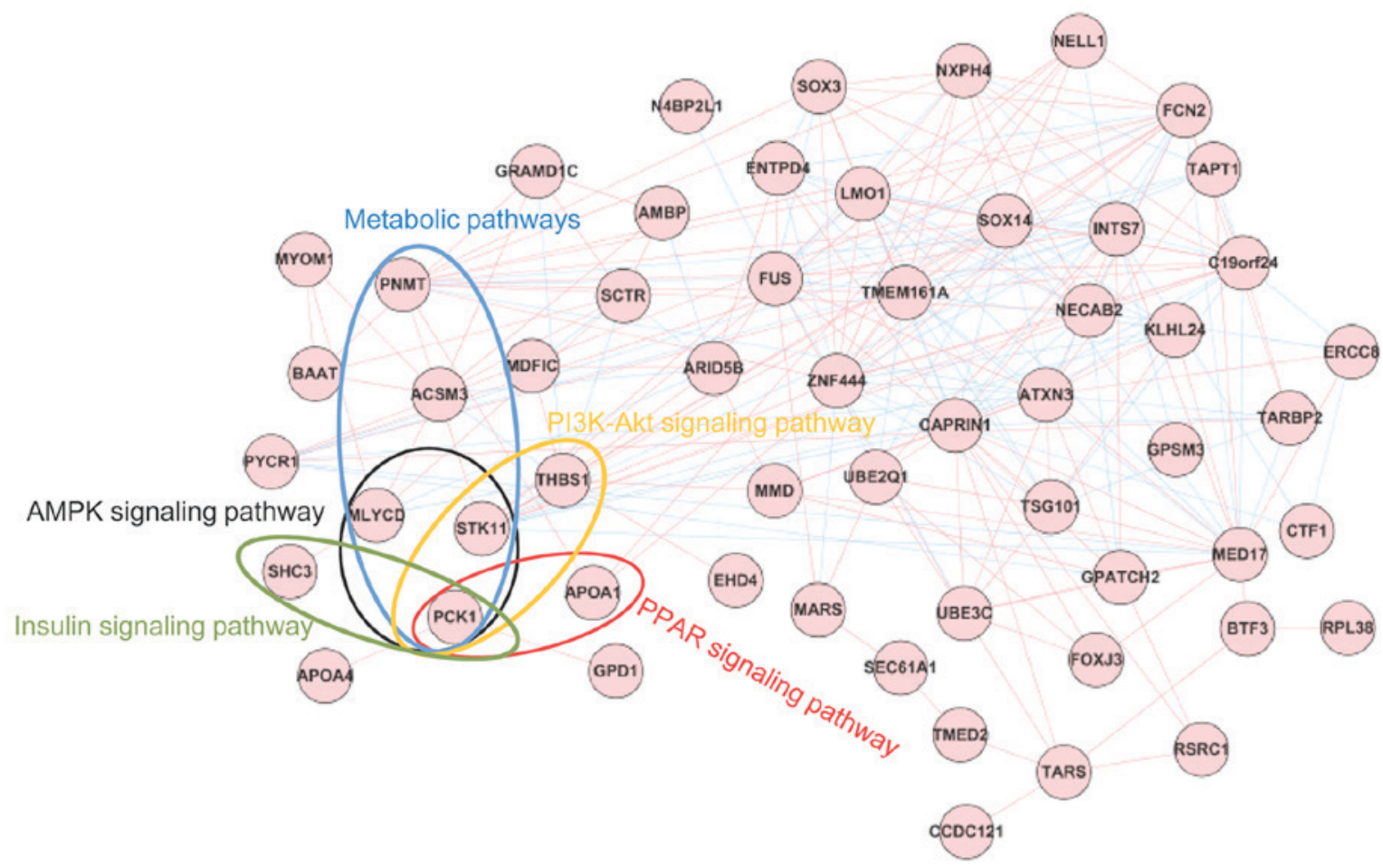

Figure 7. Co-expression network constructed for the signature genes. The signaling pathways enriched for the signature genes were merged into the network and the genes within the same circle were enriched in the same signaling pathway. AMPK, AMP-activated protein kinase; PI3K, phosphoinositide 3-kinase; PPAR, peroxisome proliferator-activated receptor. 
Table II. GO_ BP terms and signaling pathways enriched for the signature genes involved in the co-expression network.

\begin{tabular}{|c|c|c|c|c|}
\hline Category & Description & Gene no. & P-value & Gene symbol \\
\hline \multirow[t]{14}{*}{ GO_BP } & GO:0010565 regulation of cellular ketone metabolic process & 3 & 0.02095 & APOA4, MLYCD, STK11 \\
\hline & GO:0030300 regulation of intestinal cholesterol absorption & 2 & 0.023282 & APOA4, APOAI \\
\hline & GO:0046486 glycerolipid metabolic process & 4 & 0.02552 & APOA4, GPD1, APOA1, PCK1 \\
\hline & GO:0010873 positive regulation of cholesterol esterification & 2 & 0.02711 & APOA4, АРOA1 \\
\hline & GO:0046782 regulation of viral transcription & 2 & 0.02711 & $T A R B P 2, M D F I C$ \\
\hline & GO:0010872 regulation of cholesterol esterification & 2 & 0.030924 & APOA4, APOAI \\
\hline & GO:0048524 positive regulation of viral reproduction & 2 & 0.038507 & TARBP2, MDFIC \\
\hline & GO:0033700 phospholipid efflux & 2 & 0.038507 & APOA4, APOAI \\
\hline & GO:0051186 cofactor metabolic process & 4 & 0.040832 & $A M B P, G P D 1, B A A T, M L Y C D$ \\
\hline & GO:0002683 negative regulation of immune system process & 3 & 0.04189 & $A M B P, T A R B P 2, T H B S 1$ \\
\hline & GO:0044058 regulation of digestive system process & 2 & 0.042277 & APOA4, APOAI \\
\hline & GO:0034377 plasma lipoprotein particle assembly & 2 & 0.046032 & APOA4, APOAI \\
\hline & GO:0065005 protein-lipid complex assembly & 2 & 0.046032 & APOA4, АРOAI \\
\hline & GO:0007586 digestion & 3 & 0.049426 & $A P O A 4, B A A T, S C T R$ \\
\hline \multirow[t]{5}{*}{ Pathway } & AMP-activated protein kinase signaling pathway & 3 & 0.001119 & STK11, MLYCD, PCK1 \\
\hline & Peroxisome proliferator-activated receptor signaling pathway & 2 & 0.006183 & $A P O A 1, P C K 1$ \\
\hline & Metabolic pathways & 5 & 0.013991 & $\begin{array}{l}\text { ACSM3, PNMT, MLYCD, } \\
\text { PCK1, STK } 11\end{array}$ \\
\hline & Phosphoinositide 3-kinase-Akt signaling pathway & 3 & 0.017456 & STK11, THBS1, PCK1 \\
\hline & Insulin signaling pathway & 2 & 0.021115 & $S H C 3, P C K 1$ \\
\hline
\end{tabular}

GO, Gene Ontology; BP, biological process.
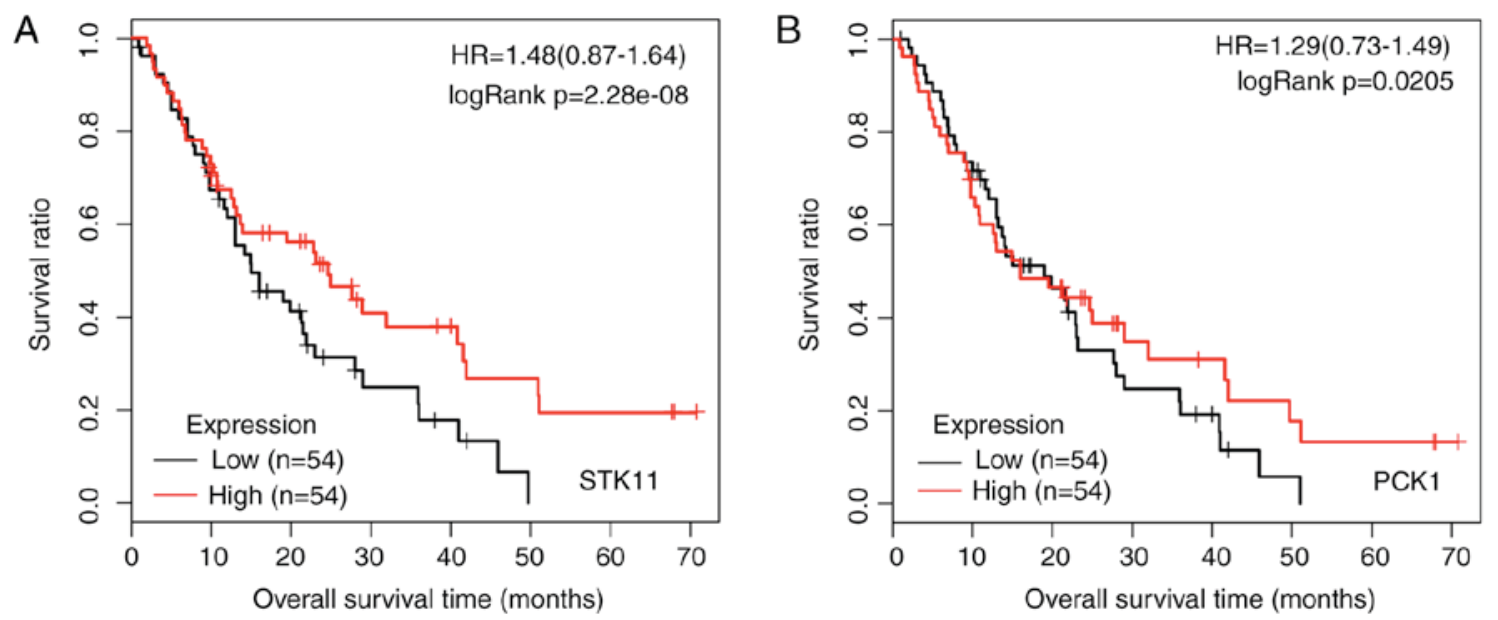

Figure 8. Kaplan-Meier survival curves for (A) STK11 and (B) PCK1. HR, hazard ratio; STK1, serine/threonine kinase 11; PCK1, phosphoenolpyruvate carboxykinase 1 .

five signaling pathways. In particular, STK11 was involved in three of the signaling pathways.

STK11/LKB1 causes somatic mutations in intraductal papillary mucinous neoplasms (IPMNs), sporadic pancreatic adenocarcinomas and biliary adenocarcinomas, and its expression is abrogated in pancreatic and biliary neoplasms $(24,25)$. Sato et al (26) identified that the STK11/LKB1 gene is associated with the development and progression of certain IPMNs (26). The LKB1-p21 axis cooperates with the Kirsten rat sarcoma viral oncogene homolog (Kras) mutation to inhibit PDAC in vivo, and downregulated $L K B 1$ may function in promoting $\mathrm{PC}$ by replacing the $p 53$ mutation $(27,28)$. As a Peutz-Jeghers syndrome gene, $L K B 1$ induces apoptosis of PC cells in a $p 73$-dependent manner (29). These studies indicate that STK11 is implicated in the prognosis of PDAC.

Stromal expression levels of THBS1 are a prognostic marker and invasive indicator in IPMN (30). By upregulating THBSI and caveolin-1 and downregulating cyclin D1, metronomic C2 and AL6 analogs perform antiangiogenic and antitumor roles in PC (31). THBS1 is implicated in cell growth and metastasis of PC cells, and stromal THBS1 immunoreactivity may be used for predicting the prognosis of PC patients (32). 
By promoting the expression of matrix metalloproteinase-9, THBS1 is important in mediating matrix remodeling in the invasion of pancreatic adenocarcinoma $(33,34)$. The signature gene, THBS1 was enriched in the PI3K-Akt signaling pathway, indicating that THBS1 may be involved in the prognosis of PDAC via the PI3K-Akt signaling pathway.

Overexpressed $B T F 3$ functions as a transcriptional regulator by mediating the transcription of tumor-associated genes in PDAC (35). RPL38, FOS-like antigen-1 and uridine phosphorylase are highly expressed in PC cell lines; therefore, they have the potential to serve as tumor markers or in tumor targeting (36). SCTR are key in regulating healthy pancreatic ductal epithelial cells, and its silence may contribute to tumor growth and progression of PC (37). SCTR is overexpressed in non-neoplastic pancreas ducts and its isoforms may be correlated with decreased secretin binding in pancreatic ductal tumors, indicating that SCTRs may represent promising clinical targets in pancreatic tumors (38). Therefore, BTF3, $R P L 38$, and SCTR may be important in the prognosis of PDAC.

There were certain limitations of the present study. The findings obtained from bioinformatics analysis require further validation via experimental studies. However, the validation experiment could not be performed in the current study due to being limited by experimental conditions and sample sources. The present results may provide valuable data for future investigations.

In conclusion, a total of 480 DEGs were identified from five datasets, and 259 prognosis-associated genes were screened from GSE28735 and GSE62452. Furthermore, the prognostic prediction system composed of 67 signature genes was constructed and validated. Notably, signature genes, including BTF3, STK11, THBS1, RPL38 and SCTR may function in the prognosis of PDAC.

\section{References}

1. Hidalgo M: Pancreatic cancer. N Engl J Med 362: 1605-1617, 2010.

2. Yadav D and Lowenfels AB: The epidemiology of pancreatitis and pancreatic cancer. Gastroenterology 144: 1252-1261, 2013.

3. Ryan DP, Hong TS and Bardeesy N: Pancreatic adenocarcinoma. N Engl J Med 371: 1039-1049, 2014.

4. Wolfgang CL, Herman JM, Laheru DA, Klein AP, Erdek MA Fishman EK and Hruban RH: Recent progress in pancreatic cancer. CA Cancer J Clin 63: 318-348, 2013.

5. Peto R, Lopez AD, Pan H and Thun MJ: World Cancer Report, 2014.

6. Mohammed S, Van Buren G 2nd and Fisher WE: Pancreatic cancer: advances in treatment. World J Gastroenterol 20: 9354-9360, 2014.

7. Dunne RF and Hezel AF: Genetics and Biology of Pancreatic Ductal Adenocarcinoma. Hematol Oncol Clin North Am 29: 595-608, 2015.

8. Yamazaki K, Masugi Y, Effendi K, Tsujikawa H, Hiraoka N, Kitago M, Shinoda M, Itano O, Tanabe M, Kitagawa Y and Sakamoto M: Upregulated SMAD3 promotes epithelial-mesenchymal transition and predicts poor prognosis in pancreatic ductal adenocarcinoma. Lab Invest 94: 683-691, 2014.

9. Masugi Y, Yamazaki K, Emoto K, Effendi K, Tsujikawa H, Kitago M, Itano O, Kitagawa Y and Sakamoto M: Upregulation of integrin $\beta 4$ promotes epithelial-mesenchymal transition and is a novel prognostic marker in pancreatic ductal adenocarcinoma. Lab Invest 95: 308-319, 2015

10. Xia S, Feng Z, Qi X, Yin Y, Jin J, Wu Y, Wu H, Feng Y and Tao M: Clinical implication of Sox9 and activated Akt expression in pancreatic ductal adenocarcinoma. Med Oncol 32: 358 , 2015 .
11. Mizuuchi Y, Aishima S, Ohuchida K, Shindo K, Fujino M, Hattori M, Miyazaki T, Mizumoto K, Tanaka M and Oda Y: Anterior gradient 2 downregulation in a subset of pancreatic ductal adenocarcinoma is a prognostic factor indicative of epithelial-mesenchymal transition. Lab Invest 95: 193-206, 2014.

12. Glazer ES, Welsh E, Pimiento JM, Teer JK and Malafa MP: TGF $\beta 1$ overexpression is associated with improved survival and low tumor cell proliferation in patients with early-stage pancreatic ductal adenocarcinoma. Oncotarget 8: 999-1006, 2017.

13. Gautier L, Cope L, Bolstad BM and Irizarry RA: Affy-analysis of Affymetrix GeneChip data at the probe level. Bioinformatics 20: 307-315, 2004

14. Smyth GK: Limma: Linear Models for Microarray Data. Springer New York, 2005.

15. Wang X, Kang DD, Shen K, Song C, Lu S, Chang LC, Liao SG, Huo Z, Tang S, Ding Y, et al: An R package suite for microarray meta-analysis in quality control, differentially expressed gene analysis and pathway enrichment detection. Bioinformatics 28: 2534-2536, 2012.

16. Porcher R: CORR insights $\left({ }^{\circledR}\right)$ : Kaplan-meier survival analysis overestimates the risk of revision arthroplasty: A meta-analysis. Clin Orthop Relat Res 473: 3443-3445, 2015.

17. Nowicka-Zagrajek J and Weron R: COR: MATLAB function to compute the correlation coefficients. Hsc Software, 2008.

18. Kohl M, Wiese S and Warscheid B: Cytoscape: Software for visualization and analysis of biological networks. Methods Mol Biol 696: 291-303, 2011

19. Reimand J, Tooming L, Peterson H, Adler P and Vilo J: GraphWeb: Mining heterogeneous biological networks for gene modules with functional significance. Nucleic Acids Res 36: W452-W459, 2008

20. Dimitriadou E, Hornik K, Leisch F and Meyer D: The e1071 package. Ethnos J Anthropol 23: 55-56, 2006.

21. Smith B, Williams J and Schulze-Kremer S: The ontology of the gene ontology. AMIA Annu Symp Proc: 609-613, 2003.

22. Aoki KF and Kanehisa M: Using the KEGG database resource. Curr Protoc Bioinformatics. Chapter 1: Unit1.12, 2012.

23. Yu G, Wang LG, Han Y and He QY: clusterProfiler: An R package for comparing biological themes among gene clusters. OMICS 16: 284-287, 2012.

24. Sahin F, Maitra A, Argani P, Sato N, Maehara N, Montgomery E, Goggins M, Hruban RH and Su GH: Loss of Stk11/Lkb1 expression in pancreatic and biliary neoplasms. Mod Pathol 16: 686-691, 2003

25. Hezel AF and Bardeesy N: LKB1; linking cell structure and tumor suppression. Oncogene 27: 6908-6919, 2008.

26. Sato N, Rosty C, Jansen M, Fukushima N, Ueki T, Yeo CJ, Cameron JL, Iacobuzio-Donahue CA, Hruban RH and Goggins M: STK11/LKB1 Peutz-Jeghers gene inactivation in intraductal papillary-mucinous neoplasms of the pancreas. Am J Pathol 159: 2017-2022, 2001.

27. Morton JP, Jamieson NB, Karim SA, Athineos D, Ridgway RA, Nixon C, McKay CJ, Carter R, Brunton VG, Frame MC, et al: LKB1 haploinsufficiency cooperates with kras to promote pancreatic cancer through suppression of p21-dependent growth arrest. Gastroenterology 139: 586-597.e1-6, 2010.

28. Wei C, Amos CI, Stephens LC, Campos I, Deng JM, Behringer RR, Rashid A and Frazier ML: Mutation of Lkbl and p53 genes exert a cooperative effect on tumorigenesis. Cancer Res 65: 11297-11303, 2005.

29. Qanungo S, Haldar S and Basu A: Restoration of silenced peutz-jeghers syndrome gene, LKB1, induces apoptosis in pancreatic carcinoma cells. Neoplasia 5: 367-374, 2003.

30. Okada K, Hirabayashi K, Imaizumi T, Matsuyama M, Yazawa N, Dowaki S, Tobita K, Ohtani Y, Tanaka M, Inokuchi S and Makuuchi H: Stromal thrombospondin-1 expression is a prognostic indicator and a new marker of invasiveness in intraductal papillary-mucinous neoplasm of the pancreas. Biomed Res 31: 13-19, 2010.

31. Bocci G, Fioravanti A, Orlandi P, Di Desidero T, Natale G, Fanelli G, Viacava P, Naccarato AG, Francia G and Danesi R: Metronomic ceramide analogs inhibit angiogenesis in pancreatic cancer through up-regulation of caveolin-1 and thrombospondin-1 and down-regulation of cyclin D1. Neoplasia 14: 833-845, 2012.

32. Tobita K, Kijima H, Dowaki S, Oida Y, Kashiwagi H, Ishii M, Sugio Y, Sekka T, Ohtani Y, Tanaka M, et al: Thrombospondin-1 expression as a prognostic predictor of pancreatic ductal carcinoma. Int J Oncol 21: 1189-1195, 2002. 
33. Qian X, Rothman VL, Nicosia RF and Tuszynski GP: Expression of thrombospondin-1 in human pancreatic adenocarcinomas: Role in matrix metalloproteinase-9 production. Pathol Oncol Res 7: 251-259, 2001.

34. McElroy MK, KaushalS, Tran CaoHS,Moossa AR, Talamini MA HoffmanRMandBouvetM:Upregulationof thrombospondin-1 and angiogenesis in an aggressive human pancreatic cancer cell line selected for high metastasis. Mol Cancer Ther 8: 1779-1786, 2009.

35. Kusumawidjaja G, Kayed H, Giese N, Bauer A, Erkan M, Giese T, Hoheise JD, Friess $\mathrm{H}$ and Kleeff J: Basic transcription factor 3 (BTF3) regulates transcription of tumor-associated genes in pancreatic cancer cells. Cancer Biol Ther 6: 367-676, 2007.

36. Sahin F, Qiu W, Wilentz RE, Iacobuziodonahue CA, Grosmark A and Su GH: RPL38, FOSL1 and UPP1 are predominantly expressed in the pancreatic ductal epithelium. Pancreas 30: 158-167, 2005.
37. Ding WQ, Cheng ZJ, McElhiney J, Kuntz SM and Miller LJ: Silencing of secretin receptor function by dimerization with a misspliced variant secretin receptor in ductal pancreatic adenocarcinoma. Cancer Res 62: 5223-5229, 2002.

38. Körner M, Hayes GM, Rehmann R, Zimmermann A, Friess H, Miller LJ and Reubi JC: Secretin receptors in normal and diseased human pancreas: Marked reduction of receptor binding in ductal neoplasia. Am J Pathol 167: 959-968, 2005.

This work is licensed under a Creative Commons Attribution-NonCommercial-NoDerivatives 4.0 International (CC BY-NC-ND 4.0) License. 